\title{
Behaviour therapies reduced urinary incontinence in older adults who were homebound and cognitively intact
}

\author{
McDowell BJ, Engberg S, Sereika S, et al. Effectiveness of behavioral therapy to treat incontinence in homebound older \\ adults.J Am Geriatr Soc 1999 Mar;47:309-18.
}

QUESTION: Do behaviour therapies reduce urinary incontinence in homebound older adults who are cognitively intact?

\section{Design}

Randomised (unclear allocation concealment), unblinded, controlled crossover trial.

\section{Setting}

Southwestern Pennsylvania, USA.

\section{Participants}

105 adults $\geq 60$ years of age (mean age $77 \mathrm{y}, 91 \%$ women, $93 \%$ white) who had nurse confirmed urinary incontinence ( $\geq 2$ urinary accidents/wk and incontinence persisting for $\geq 3 \mathrm{mo}$ ), met the Health Care Financing Administration criteria for being homebound, and were cognitively intact (Folstein Mini Mental State Examination score $\geq 24$ ). Participants had a mean of 8 medical problems and 53\% had functional limitations. Participants were identified and referred by homecare nurses. Exclusion criteria were severe pelvic inability to toilet independently, or inability to provide satisfactory self report of bladder diary data. Follow up

\section{Intervention}

After 2 weeks of baseline bladder diaries, 53 participants were allocated to the treatment group and 52 were allocated to the control group. Participants in the treatment group received behaviour therapies (biofeedback assisted pelvic floor muscle exercises, urge and stress strategies, and bladder retraining) during 8 weekly nurse practitioner home visits. Each session lasted 40-60 minutes. Nurses adjusted the protocol based on the participant's progress. Participants in the control group had an 8 week observation period; nurse practitioners made 30 minute home visits every 1-2 weeks to provide social interaction. Control participants then completed 2 weeks of bladder diaries and received the treatment protocol. All participants completed 2 weeks of posttreatment bladder diaries.

\section{Main outcome measure}

Self reported urinary accidents (bladder diaries).

\section{Main results}

Participants who received behaviour therapies had a lower median number of urinary accidents/day $(0.7 \mathrm{v}$ $2.7, \mathrm{p}<0.001)$ and a greater median per cent reduction in urinary accidents/day $(75.0 \% v 6.4 \%, \mathrm{p}<0.001)$ than participants in the control group. prolapse, terminal illness, post-void residual $>100 \mathrm{ml}$, was $89 \%$.

\section{Conclusion}

Behaviour therapies that included biofeedback assisted pelvic floor muscle exercises, urge and stress strategies, and bladder retraining were effective in the short term reduction of urinary incontinence in older adults who were homebound and cognitively intact.

\section{COMMENTARY}

The authors note that urinary incontinence affects 13-53\% of homebound older adults. It has a substantial impact on quality of life, healthcare costs, and rates of institutionalisation of elderly people. ${ }^{1}$ Effective treatment of urinary incontinence is an important healthcare issue.

McDowell et al found an improvement in urinary incontinence 2 weeks after completion of an 8 week behaviour therapy intervention. This short term improvement is consistent with the findings of a systematic review and studies published since the review. ${ }^{2-4}$ The authors conducted long term follow up every 3 months for 1 year to monitor continence status and adherence to treatment recommendations, but these results were not included in this report. It will be interesting to see these results when they are published.

The strengths of this study are the 30 minute weekly attention placebo given to the control group over the first 8 weeks and the crossover design in which participants in the control group crossed over and received the behaviour therapies after the initial 8 week period.

The clinical utility of this study is substantial for nursing practice. The behaviour techniques fall within a nurse's scope of practice. The study clearly identified the strategies used: pelvic floor muscle exercises, stress and urge training, and bladder training. Biofeedback was used to teach participants the correct method of contracting pelvic floor muscles. Nurses would need the necessary knowledge, skill, and judgment to implement these techniques. Behaviour therapy is not recommended for treatment of overflow urinary incontinence. ${ }^{1}$ I would recommend behaviour therapy for homebound older adults with non-overflow urinary incontinence, but would monitor the long term effects.

Lisa Hamilton, RN, MSc Clinical Nurse Specialist Geriatric Rehabilitation Toronto Rehabilitation Institute Toronto, Ontario, Canada

1 Fantl JA, Newman DK, Colling J, et al. Urinary incontinence in adults: acute and chronic management. Clinical practice guideline no 1. Rockville, MD: Agency for Health Care Policy and Research, 1996.

2 Roe B, Williams K, Palmer M. Bladder training for urinary incontinence (Cochrane Review, latest version 26 Aug 1998). In: Cochrane Library. Oxford: Update Software.

3 Burgio KL, Locher JL, Goode PS, et al. Behavioral vs drug treatment for urge urinary incontinence in older women: a randomized controlled trial. JAMA 1998;280:1995-2000.

4 Wyman JF, Fantl JA, McClish DK, et al. Comparative efficacy of behavioral interventions in the management of female urinary incontinence. Am J Obstet Gynecol 1998;179:999-1007.

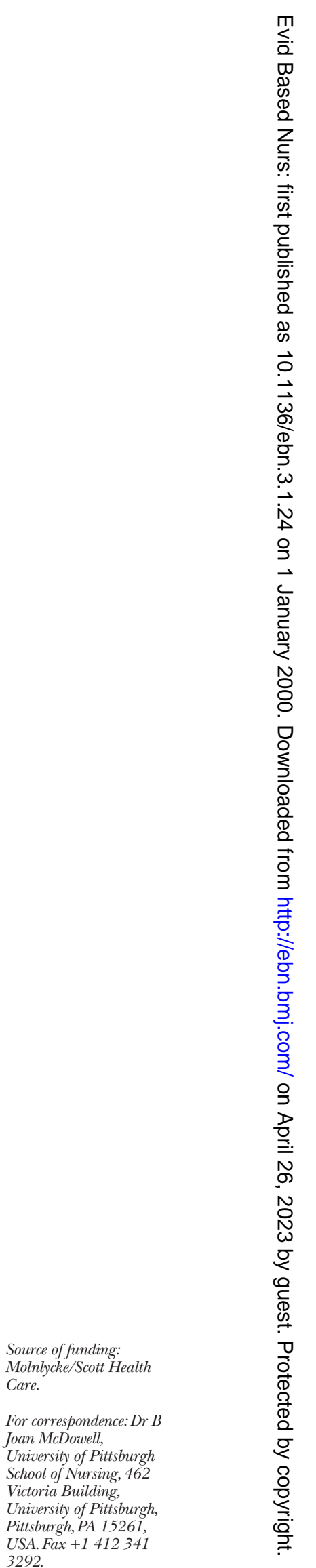

